\title{
Equipment body feeling maintenance teaching system Research Based on Kinect
}

\author{
Fushuan $\mathrm{Wu}^{1, \mathrm{a}}$, Jianren Wang ${ }^{1, \mathrm{~b}}$, Chen $\mathrm{Mei}^{1, \mathrm{c}}$, Yiguang Xiong ${ }^{2, \mathrm{~d}}$, \\ ${ }^{1}$ Wuhan Mechanical Technology College, Wuhan, 430075, China \\ ${ }^{2}$ Kunming college for ethnic cadres, Shifang Sichuan, 618400, China

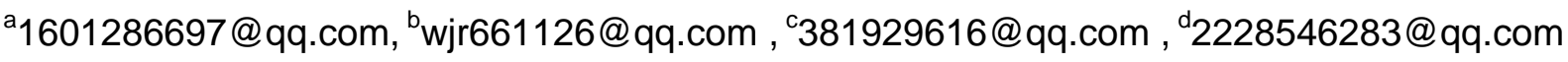

Keywords: Body feeling; Kinect; Virtual maintenance.

\begin{abstract}
Based on the virtual maintenance teaching and motion-sensing technology, studied the introduction of the feeling device Kinect, operation substitute design, Maintenance of posture position etc. under the Unity3D development environment. Practical application proved that body feeling maintenance teaching system is a more superior mode of training than traditional keyboard mouse operation.
\end{abstract}

\section{Introduction}

Due to the complex structure, high price of modern equipment, the fewer teaching equipment, and using real equipment teaching easily cause safety accident, so the virtual maintenance teaching system has become an important supplementary means of equipment maintenance training. However, because of the limitation of the human-computer interaction means, a lot of virtual maintenance teaching system are still using traditional keyboard mouse as interactive devices. In virtual maintenance training, generally only can through the mouse click the tools icon representing picked up his tools, and then use the mouse click operation object to play decomposition combined with animation, actually reach the effect of training is familiar with the decomposition process, and the name of the tool, and the use methods of specific tools are difficult to reflect, or "hands-on" ability is very difficult to cultivate. But often students lacking is the practical ability, it is more easily fall into trouble when facing a practical maintenance classroom. The emergence of the body feeling equipment device Kinect, opens up a new path for the virtual maintenance teaching system.

Kinect body feeling equipment is a somatosensory operating equipment developed by Microsoft for game XBOX360 , relatively quick past \$one million worth of optical motion capture system, the cost is very low, of course, the identification accuracy is relatively low, but it is enough to be able to do the game, interface operation and other basic requirements. Besides real-time dynamic capture ability, at the same time, it also has image recognition, speech recognition and other practical functions, is a promising body feeling equipment is. Currently the body feeling game and application using the Kinect as external equipment has reached hundreds of applications including robot visual identification, modeling business selling machines, modeling of three-dimensional scanning and so on. In the field of education, the application of Kinect in the interactive electronic whiteboard is also studied.

\section{The Kinect and Its Development}

Kinect's appearance is shown in figure 1, is mainly composed of CMOS infrared emission and sensors, RGB camera and microphone. Its core technology comes from Israel PrimeSense Company's low-cost three-dimensional detection chip and Microsoft research of human action recognition system. PrimeSense Company once serviced for Israel's military, their chip and a complete set of software is a good way to deal with three-dimensional space coordinate information of the object. 


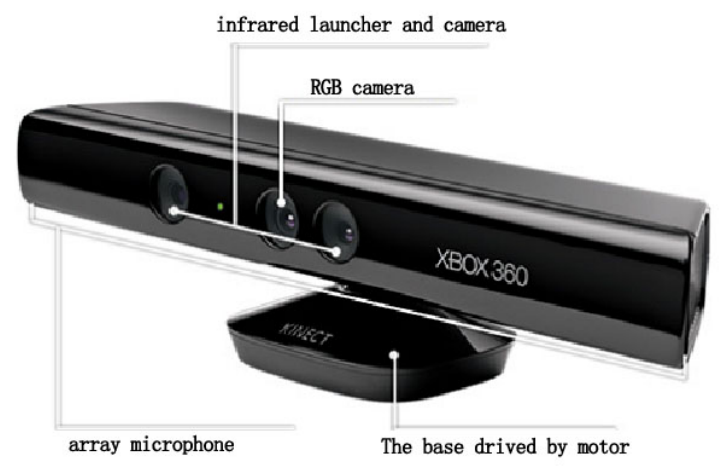

Figure 1 Kinect appearance

CMOS infrared sensor by encoding light emission depth image, pure black represents infinity, both in pure white represents the zero distance, with the concentration of grey represents distance. Sensors at 30 frames per second generation depth of field image flow. Chips will dig out from the depth of field image and body image was introduced into the Exemplar artificial intelligence processing system, the system is a large amount of experience accumulated through image matching, generates a 20 joints skeleton system (see figure 2). In the middle of Kinect, the RGB camera captures the human body image map, in addition to helping equipment to detect the location of the user, it can also be used to take photos, in the base of device there is the driver plastic gear motor, used for automatic correction cameras and space sensors, making the Kinect get the best position to observe users.

At present, the secondary development kit of Kinect has Microsoft access for Windows SDK [2] and Prime Sense OpenNI [3]. Both have their own strengths, and developers can choose according to the demand. OpenNI provides Unity3D plugin package directly, so we chose the OpenNI as development tool.

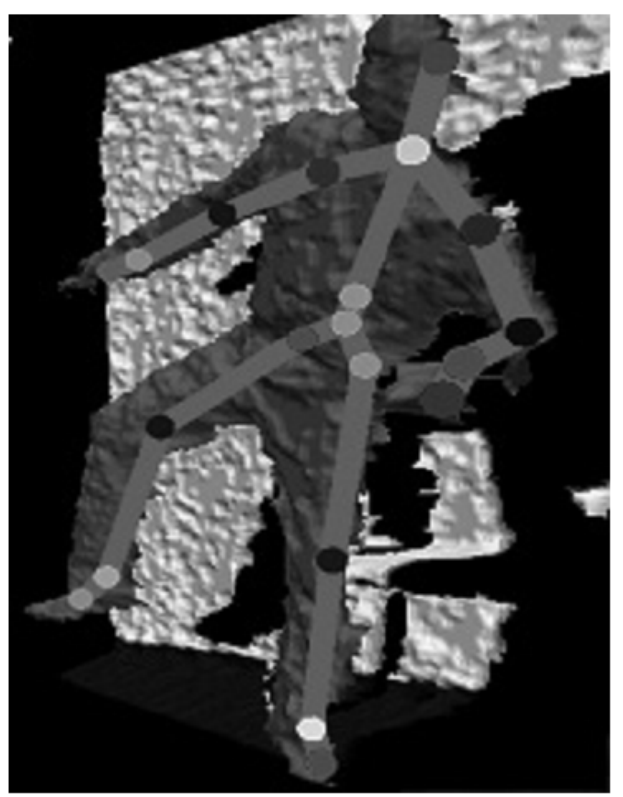

Figure2 Skeleton diagram of two top-generated forms

\section{Equipment Somatosensory Maintenance Teaching System}

1) Introduction of hardware and software

Under the OpenNI framework, to make maintenance training system to be able to use Kinect, you will first need to install OpenNI (version for 1.5.2.23), NITE middleware (version for 1.5.2.21) 
responsible for the analysis of the image data and driver module SensorKinect (version for 5.1.0.25). Virtual interactive software using Unity3D4.0, for direct use to access data, introduces OpenNI_Unity_Toolkit - 0.9.7.4 plug-in package. The package offered Unity ability to directly access OpenNI compatible sensor. The hardware adopted a Kinect for XBOX360 external power adapter and the remote control lever. In the introduction of plug-in package, Unity3D can form OpenNI fabrication and script (see figure 3). Users can directly drag the fabrication and scripts to use in the scene, can do not need to care about the underlying API and directly use "drag-put" way to use the data.

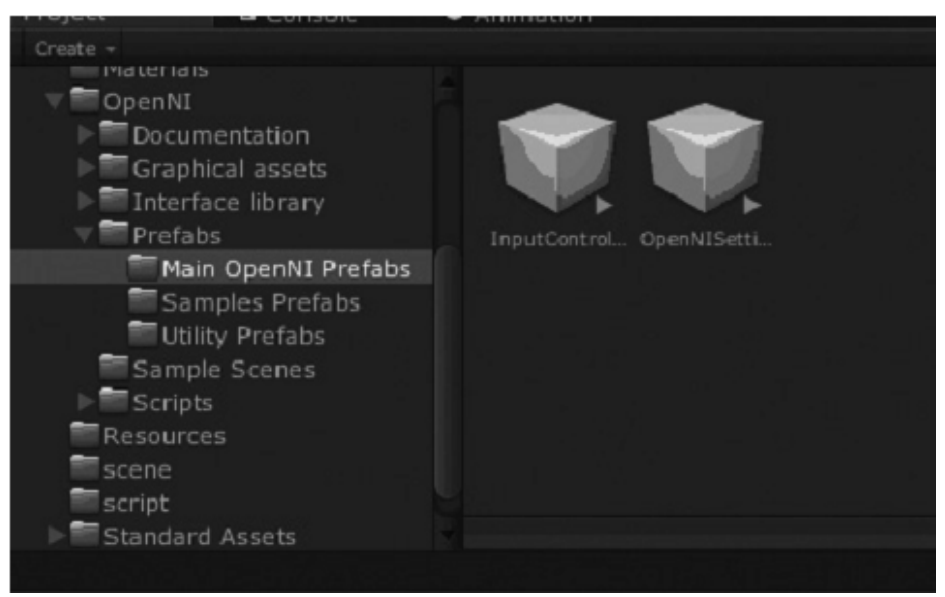

Figure 3 OpenNI precast

2) Introduction of body feeling operation

The precision of the Kinect is limited, so using body feeling operation in virtual maintenance, the more appropriate way is introduce into a virtual human in virtual scene, and then passed the operator body data captured by Kinect to the virtual human body, making the virtual synchronous movement between virtual human and operators, and operators by judging the collision of virtual people and objects in the scene to determine the interaction of timing. If directly through the Kinect to operate interface element, there could be beating phenomenon, which would leads to interactive feeling bad. Virtual human production can be produced by 3dmax and other software, bone skin of human body model into the Unity again, and then in the heart of the Unity of virtual human bones and OpenNI provides access data matching Joints, the specific approach is to set up NISkeleton Joints in the Controller script to control array (as shown in figure 4). After bone matching relationship established, in a virtual hand set bounding box collision, the bounding box contains the whole hand. So in the program can through the capture of collision events, make the operator through the virtual people take put tools, parts and components, operation implement decomposition combining virtual maintenance.

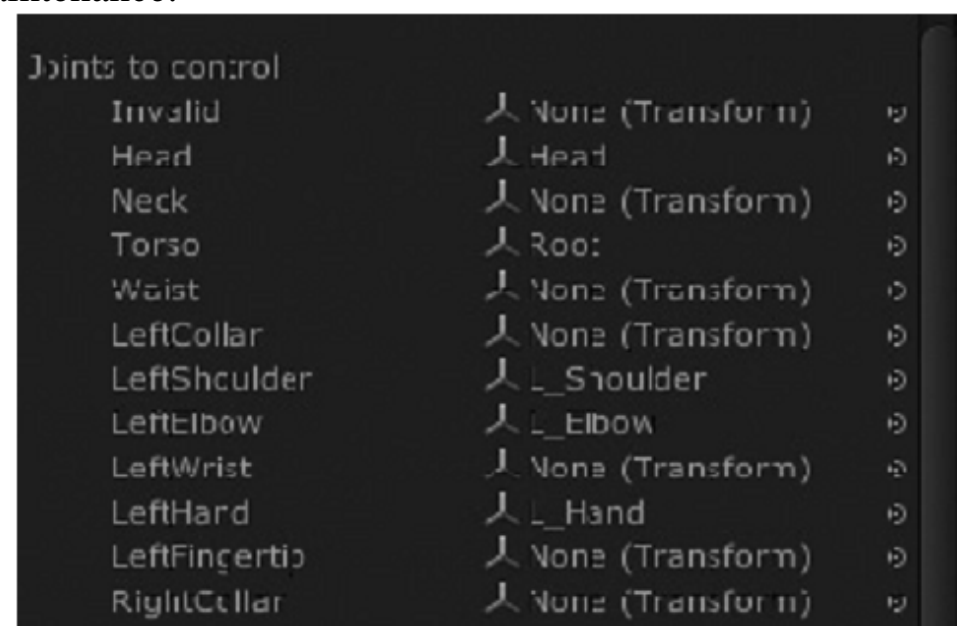

Figure 4 rigging array

3) Introduction of body feeling operation

Maintenance work is done through a series of processes and equipped with basic training, and 
can be resolved as big combination and decomposition of parts combined with homework. In order to form a generalization of the system structure combined with the need to provide in the form of text decomposition process, the program runs according to the chosen maintenance subjects, read the corresponding process and model can implement decomposition combined with training. The definition of maintenance process use Office Project 2003, which can easily establish PERT process. And then export the text file for maintenance training system calls. At each step in the process it is necessary to define operator action, in order to facilitate preparation of action and gesture judgment, and a flexible attitude definition format is proposed. Format is composed of a series of short sentences, between each phrase to ";" separated, in the form of "LArm; RArm; LHip; RHip, Spine, Picture". Format is similar to a single phrase, such as the RArm section, a complete definition for "RArm: wrist position @ parts name", including "wrist position" is a vector x, y, z, numerical to virtual human right shoulder department as a starting point, the body for the $\mathrm{z}$ axis positive, the right to $\mathrm{x}$ axis and head up to $\mathrm{y}$ axis, to arm length is 1.0 , the relative position accurate to one decimal places. Such as "1,0,0" is to the right unbend arm on behalf of the level. People in the heart of the application of virtual hand and shoulder position directly in the form of parameters passed in, can get real-time hand relatively shoulder joint relative coordinates, through the normalized processing is obtained with the wrist position.

RightArmLength (the program is worked out in advance of virtual human right arm length).

Vector3 hp=rHand.position-rArm.position;

$\mathrm{hp}=\mathrm{hp} /$ rightArmLength;

Then wrist location and requirements is presupposed, get the attitude deviation vector (handF). For consideration $\mathrm{X}, \mathrm{Y}, \mathrm{Z}$ three directions deviation at the same time , take the attitude deviation vector length to measure. Considering the action shall permit the operator has a certain error, this paper set the allow value (tolerence) to 0.4 , can ensure that the operator attitude is correct, at the same time not too strictly.

float hadF=(handpos-hp).magnitude;

if(hadF $<$ tolerence)

ifRight=ifRight*1;

else

$\{\quad$ ifRight=ifRight*0;

return false; $\}$

Phrase "part name" items represents hand or hand tools and the parts are in the intersection position, meaning that when the action is done, need the hand or the hand tool touch the parts. For example when the right hand picks off the bottom cover screw, the left hand should hold bottom cover to prevent falling.

Through the study of the definition of each body part, so can request when the personnel to perform some sort of decomposition action in the virtual training.

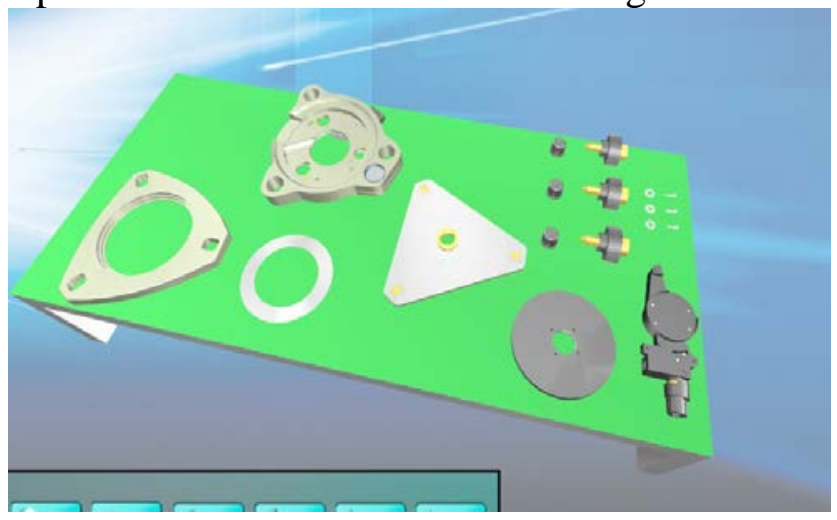

Figure 5 interface diagram of body sense virtual maintenance training system

3) Instance profile

A portion of the decomposition training is a key subject that students have to practice, as a object, establish the sense of body type decomposition combining with maintenance training system. 
Program developed by Unity3D 4.0, set up a virtual scene, and then introduced the virtual people, binding camera on the head of the virtual human , can observe from the Angle of double three-dimensional scene (as shown in figure 5).

Through the Kinect, the operator can control the virtual people walking around in the scene, to access a variety of tools on the table, put tools on tool table, left and right hand exchange tool, using hand tools or hand to interact with parts, combining sequential decomposition step by step according to the decomposition, decomposition of specific parts need to set the right attitude to decomposition. Use subjective feelings strong immersion, smooth operation, tool using simulation.

\section{Summary}

The body feeling operation is one of the future focus directions of the human-computer interaction. Equipment body feeling maintenance teaching system give full play to the advantages of the technique on the body feeling, can effectively cultivate students' practical ability, improve the efficiency of teaching, is a very good way of teaching. Although at present due to the restrictions on the accuracy of the device, motion capture has delay and jitter shortcomings, but the body feeling technology improves quickly. Kinect2 generation is reported to the forthcoming, some other body feeling equipment with high precision will also be available. All in all, introducing the advanced motion-sensing technology Kinect represents in a timely manner into virtual maintenance training, which will play a positive significance in improving the level of maintenance training.

\section{References}

[1] Zhenguo Liu, Fu Jiang, Bin Shen, Tao Wang,Xin Yu,Wensheng Huang. Virtual Laboratory for Equipment Repair based on VIRTOOLS [J] .Applied Mechanics and Materials,ISSN:1662-7482.

[2] Hu Yong Naikun Wei,Dianliang Wu, Xiumin Fan. Assembly design based on the collaborative virtual environment system [J]. Computer engineering, 2010.6.

[3] D.Tian and N.Georganas.A coverage-Preserving Node Scheduling Scheme for Large Wireless Sensor Networks. 2002, 9.

[4] Runfang Chang, Aihua Zhang. Immersion promotion strategy research [J], 2011 (4).

[5] Castro M., Lopez-Rey, A., Perez-Molina C.M., Colmenar A., de Mora C., Yeves F., Carpio J., Peire J., Daniel J. S., “Examples of distance learning projects in the European Community”, IEEE Transactions on Education, Vol. 44, Issue 4, Nov. 2001 pp.406 - 411.

[6] Li Qin. Morphological characteristics of immersion transmission study [J]. Modern communications, 2013 (2).

[7] Deshpande, S. G., Jenq-Neng Hwang, “A real-time interactive virtual classroom multimedia distance learning system”, IEEE Transactions on Multimedia, Vol 3, Issue 4, Dec. 2001 pp. 432 $-444$.

[8] Guowei liang, Yuan Bo. Theory of virtual reality technology to create network immersion interactive art space [C]. Academic exchanges, 2010.12.

[9] Karim Yaghmour, “Building Embedded Linux Systems”, 2003 O'REILLY.

[10] Caili Bu, Hongmin Zhao. Immersion theory application in the network study [J]. Journal of software review, 2013 (9). 